Objectives: To assess the frequency of depression and anxiety in patients with Familial Mediterranean Fever (FMF)

Methods: In this study, 77 FMF patients aged 18 and over who were followed up in Sakarya University Education and Research Hospital, Department of Rheumatology, and 78 healthy volunteers aged 18 and over as thecontrol group. Beck depression scale and Beck anxiety scale were used to depression and anxiety, respectively. Beck'sdepression scale was evaluated as 9 and below normal, 10-16 mild depression, 17-29 moderate depression, 30-63 severe depression. Beck anxiety scale was evaluated as 0-8 normal, 8-15 mild anxiety, 16-25 moderate anxiety, 26 and above severe anxiety.FMF disease severity was determined by Pras scoring.

Results: The study group, comprised 77 diagnosed with FMF with a meanage of 37.18 and a control group comprised of 78 healthy controls $(C)$ with a meanage of $35.32(p=0,058)$. In studygroup $(P) \% 63.6$, control group $(C) \% 53.8$ as female. $\% 36.4$ of thestudy group $(C), \% 46.2$ of the control group are male. $(p=0,216)$. The prevalence of depression was significantly higher in FMF patients compared to the control group (in order P;C: normal \%24,7; \%47,4, mild depression: \%40.3; $\% 26.9$, moderate depression \%26; \%19.2, severe depression $\% 11.7 ; \% 6.4$ $\mathrm{p}<0.015)$. Similarly in depression results; the prevalence of anxiety was significantly higher in FMF patients compared to the control group (in order $\mathrm{P} ; \mathrm{C}$ normal $\% 23,4 ; \% 57.7$, mild anxiety \%26; \%20.5, moderate anxiety $\% 26 ; \% 15.4$, severe anxiety \%24.4; \%6.4 $p<0,001)$. Depression status was not correlated with FMF disease severity $(p=0.645)$. A correlation was found between FMF severity and anxiety which it is which was found statistically significant $(p=0.005)$. There was no relationship between erythrocyte sedimentation rate and C-reactive protein with depression and anxiety.

Conclusion: Both anxiety and depression frequency are increased in FMF patients compared to healthy controls.

References:

[1] Livneh A, Langevitz P, Zemer D et al. (1997) Criteria for the diagnosis of familial Mediterranean fever. Arthritis Rheum 40 (10), 1879-85.

[2] Alonso J, Ferrer M, Gandek B, Ware JE Jr, Aaronson NK, Mosconi P, Rasmussen NK, Bullinger M, Fukuhara S, Kaasa S, Leplège A, IQOLA Project Group (2004) Health-related quality of life associated with chronic conditions in eight countries: results from the International Quality of Life Assessment (IQOLA) Project. Qual Life Res 13:283-298

[3] Makay B, Emiroglu N, Unsal E (2010) Depression andanxiety in children and adolescents with familial Mediterranean fever. Clin Rheumatol 29, 375-9.

[4] Giese A, Ornek A, Kilic L, Kurucay M, Sendur S. N., Lainka E, Henning B. F. Anxiety and depression in adult patients with familialMediterranean fever: a study comparing patients living in Germany and Turkey. International Journal of Rheumatic Diseases 2017; 20: 2093-2100

Disclosure of Interests: None declared

DOI: 10.1136/annrheumdis-2020-eular.5852

\section{AB1035 INTESTINAL MICROBIOTA COMPOSITION OF ADULT PATIENTS WITH FAMILIAL MEDITERRANEAN FEVER AND HEALTHY CONTROLS (THE RHEUMA-BIOTA STUDY)}

N. S. Yasar Bilge ${ }^{1}$, V. Perez Brocal ${ }^{2}$, T. Kașifoğlu ${ }^{1}$, U. Bilge ${ }^{3}$, N. Kasifoglu ${ }^{4}$, A. Moya ${ }^{5,6}$, E. C. Dinleyici ${ }^{7} .{ }^{1}$ Eskisehir Osmangazi University Faculty of Medicine, Department of Internal Medicine, Division of Rheumatology, Eskişehir, Turkey; ${ }^{2}$ Fundación para el Fomento de la Investigación Sanitaria y Biomédica de la Comunitat Valenciana, Valencia, Spain; ${ }^{3}$ Eskisehir Osmangazi University Faculty of Medicine, Department of Family Medicine, Eskişehir, Turkey; ${ }^{4}$ Eskisehir Osmangazi University Faculty of Medicine, Department of Microbiology, Eskişehir, Turkey; ${ }^{5}$ Integrative Systems Biology Institute (I2Sysbio); University of València and Spanish Research Council (CSIC), Valencia, Spain; ${ }^{6}$ Foundation for the Promotion of Sanitary and Biomedical Research of Valencian Community (FISABIO), Valencia, Spain; ${ }^{7}$ Eskisehir Osmangazi University Faculty of Medicine, Department of Pediatrics, Eskişehir, Turkey

Background: Although Familial Mediterranean Fever (FMF) is a monogenic disease, microbiota composition may play role in the pathogenesis or phenotypic expression.

Objectives: We aim to evaluate the intestinal microbiota composition in patients with FMF and to compare with healthy controls.

Methods: In this prospective cohort study, a group of 10 adult patients with FMF and 10 age-appropriate healthy controls, for which there was strict inclusion/ exclusion, were enrolled. Fecal samples were stored at $-80^{\circ} \mathrm{C}$ until DNA extraction. A region of the 16S rRNA gene (V3-V4) was selected and sequencing was performed on the Illumina MiSeq platform at the Sequencing and Bioinformatics Service of FISABIO foundation.

Results: Alpha and beta diversity tests were similar between FMF and control groups except that Chao1 index. Chao1 index was modestly decreased in FMF group comparing the healthy controls $(p<0.05)$. Our results showed differences in the intestinal microbiota composition of patients with FMF, with a higher abundance of Eggerthella, at genus level. At species level, Eggerthella sinensis and Eggerthella lenta were more abundant in patients with FMF.

Conclusion: Eggerthella lenta was previously shown to be higher in type II diabetes, multiple sclerosis, rheumatoid arthritis and some disseminated infections. In this study we firstly showed abundance of Eggerthella in patients with FMF especially in $E$. sinensis and E. lenta; in addition to. Whether any of observed associations are causal, or the direction of causality is unclear yet and further studies with patients with FMF at the first diagnosis might clarify this issue.

Disclosure of Interests: None declared

DOI: 10.1136/annrheumdis-2020-eular.3906

\section{AB1036 CLINICAL MANIFESTATIONS, CLINICAL COURSE, AND OUTCOMES OF IMMUNOGLOBULIN G4 RELATED DISEASE}

W. Katchamart ${ }^{1}$, K. Phaopraphat ${ }^{2}$, P. Ngamjanyaporn ${ }^{3}$, P. Narongroeknawin ${ }^{4}$, N. Kasitanon ${ }^{5}{ }^{7}$ Division of Rheumatology, Department of Medicine, Faculty of Medicine Siriraj Hospital, Mahidol University, Bangkok, Thailand, Bangkok, Thailand; ${ }^{2}$ Department of Medicine, Faculty of Medicine Siriraj Hospital, Mahidol University, Bangkok, Thailand, Bangkok, Thailand; ${ }^{3}$ Division of Rheumatology, Department of Medicine, Faculty of Medicine, Ramathibodi Hospital, Mahidol University, Bangkok, Thailand, Bangkok, Thailand; ${ }^{4}$ Division of Rheumatology, Department of Medicine, Faculty of Medicine Phramongkutklao Hospital, Phramongkutklao College of Medicine, Bangkok, Thailand, Bangkok, Thailand; ${ }^{5}$ Division of Rheumatology, Department of Medicine, Faculty of Medicine, Chiang Mai University, Chiang Mai, Thailand, Chiang Mai, Thailand

Background: Immunoglobulin G4 related disease (IgG4-RD) is an uncommon chronic systemic autoimmune disease, pathologically characterized by lymphoplasma cell, IgG4 plasma cell or storiform fibrosis infiltration with elevated serum IgG4 level. IgG4-RD is a new disease and not widely recognized.

Objectives: The aim of this study was to describe clinical manifestations and outcomes of IgG4-RD in Thai patients

Methods: This multicenter retrospective cohort study included patients who aged $\geq 18$ years and were diagnosed with IgG4-RD according to $2011 \mathrm{com}-$ prehensive or consensus diagnostic criteria, between 2000 and 2019 in four academic centers in Thailand. Baseline characteristic, laboratory and pathologic findings, treatments, and outcomes were systematically reviewed.

Results: Of the 110 patients included, $71 \%$ were male with mean age (SD) of 59.6 (13.3) years and median disease duration (IQR) of 28.8 (14.6-53.5) months Single organ involvement was observed in 60 patients $(54.5 \%)$. The most common presenting organ involvement was the orbit $(29 \%)$, followed by the salivary glands (19\%), lacrimal glands (18\%), bile duct (16\%), and pancreas $(11 \%)$. The most frequently affected organs were the orbits (34\%), followed by the salivary glands (26\%), lacrimal glands (20\%), bile duct (19\%), and lymph nodes (19\%) Ninety-six percent $(96 \%)$ had lgG4 level of more than $135 \mathrm{mg} / \mathrm{dl}$ at presentation. Most patients (92\%) were treated with corticosteroid (CS) alone or in combination with immunosuppressive agents. Azathioprine (47\%) and methotrexate $(11 \%)$ were the most commonly used immunosuppressive agents. Additionally, $20 \%$ required surgery, and $6.4 \%$ underwent stent insertion. One-fourth (26\%) were in remission with successfully CS tapering, while $37 \%$, and $29 \%$ had complete, and partial response. Nevertheless, $22 \%$ relapse with median time to relapse (IQR) of $22.2(12.8-41.1)$ months. Relapse was common in patients with orbital ( $p=0.001)$ and lung $(p=0.007)$ involvement, and patients with longer disease duration (median 44.1 and 23.1 months, $P=0.001$ ), while serum IgG4 level was insignificantly higher in relapse group (median $1,085 \mathrm{vs} .850 \mathrm{mg} / \mathrm{dL}, \mathrm{p}=0.28$ ). Conclusion: IgG4-RD is a chronic systemic autoimmune disease with diverse manifestations, response to treatment, and outcomes. Most patients responded well to CS and immunosuppressive agents with notable relapse rate, while minority required surgery or mechanical intervention.

References:

[1] Wallace ZS, Zhang Y, Perugino CA, Naden R, Choi HK, Stone JH. Clinical phenotypes of IgG4-related Disease: an analysis of two international cross-sectional cohorts. Ann Rheum Dis. 2019;78(3):406-12.

[2] Martinez-Valle F, Fernandez-Codina A, Pinal-Fernandez I, Orozco-Galvez O, Vilardell-Tarres M. IgG4- related disease: Evidence from six recent cohorts. Autoimmun Rev. 2017;16(2):168-72

Acknowledgments: None

Disclosure of Interests: None declared

DOI: 10.1136/annrheumdis-2020-eular.1206

\section{AB1037 CANAKINUMAB FOR TREATMENT OF ADULT ONSET STILL'S DISEASE-RESULTS OF THE 24 WEEKS TREATMENT AND BEYOND: A MULTI-CENTRE, PLACEBO-CONTROLLED STUDY (CONSIDER)}

C. Kedor ${ }^{1}$, J. Listing ${ }^{2}$, J. Zernicke ${ }^{1}$, A. Weiß ${ }^{2}$, F. Behrens ${ }^{3}$, N. Blank ${ }^{4}$, J. Henes ${ }^{5}$, J. Kekow ${ }^{6}$, A. Rubbert-Roth ${ }^{7}$, H. Schulze-Koops ${ }^{8}$, E. Seipelt ${ }^{9}$, C. Specker ${ }^{10}$, 